\title{
Utility of polygraphic studies for sleep apnea screening in the setting of tertiary care hypertension outpatient clinic
}

\author{
Maria Nowak, Anna Komand, Dawid Ostrówka, Marta Jancewicz, Eliza Miszkowska-Nagórna, \\ Anna Szyndler, Jacek Wolf, Krzysztof Narkiewicz
}

Department of Hypertension and Diabetology, Medical University of Gdansk, Gdansk, Poland

\begin{abstract}
Background: Prevalence of obstructive sleep apnea (OSA) in patients with hypertension outnumbers the prevalence reported in the general population. Concurrently, majority of patients remain undiagnosed. Given the fact that untreated OSA contributes to ineffective hypotensive treatment, and higher rate of complications is ascribed to hypertension, early OSA diagnosis and its elimination constitutes one of the key clinical goals. Polysomnography comprises a golden standard in sleep apnea diagnosis, however it is a time-consuming and expensive procedure which requires hospitalization. Therefore, we assessed the utility of simplified polygraphic (PG) studies in the OSA screening of patients with hypertension requiring regular tertiary care.

Material and methods: The study was conducted in the Outpatient Tertiary Care Clinic of the Medical University Hospital of Gdansk. We enrolled consecutive patients regardless of OSA symptoms presence ( $\mathrm{n}=243)$, as well as patients with OSA suspicion ( $\mathrm{n}=75)$. A total of 318 patients $(39 \%$ females) were subjected to ambulatory, onenight polygraphic study (ApneaLink ${ }^{\mathrm{TM}}$ ). The following signals were recorded: airflow (pressure cannula), respiratory movements, and pulse oximetry. Additionally, anthropometrics, Epworth Sleepiness Scale (ESS), selected clinical symptoms, and drug-regimen were recorded.

Results: Three hundred and eight (97\%) patients completed the study (10 dropouts due to study failure). Patients were $57.7 \pm 11.5$ years old, had BMI $=30.0 \pm 5.0 \mathrm{~kg} / \mathrm{m}^{2}$, and average ESS $=5.7 \pm 4.6 .65 .3 \%$ of all patients were characterized by AHI $\geq 5$ (62.2\% vs. $74.7 \%$ in Random vs. Symptomatic group, respectively; $\mathrm{P}=0.49)$, whereas $27.6 \%$ of patients were diagnosed with AHI $\geq 15$ ( $23.6 \%$ in Random group and $40.0 \%$ in Symptomatic group). In logistic regression analysis, nocturia $(>2)$ increased the odds of AHI $\geq 30$ diagnosis approximately 3.5 times in a fully adjusted model.

Conclusions: Our results justify routine polygraphic screening of hypertensive patients requiring tertiary care. Abridged medical history may substantially increase the odds for positive screening.

key words: polygraphy, nocturia, hypertension, sleep apnea
\end{abstract}

Arterial Hypertens. 2016, vol. 20, no. 1, pages: 5-10

DOI: $10.5603 /$ AH.2016.0002

\section{Introduction}

Prevalence of obstructive sleep apnea (OSA) in cardiovascular patients especially with hypertension difficult to control outnumbers the prevalence reported in the general population $[1,2]$. Concurrently, ma- jority of patients remain undiagnosed. Given the fact that untreated OSA contributes to ineffective hypotensive treatment, and higher rate of complications is ascribed to hypertension, early OSA diagnosis and its elimination constitutes one of the key clinical

Address for correspondence: Jacek Wolf, MD

Dept. of Hypertension and Diabetology, Medical University of Gdansk

Dębinki 7C str., 80-952 Gdansk; e-mail: lupus@gumed.edu.pl 
goals. Polysomnography (PSG) constitutes a golden standard in sleep apnea diagnosis, however, it is a time-consuming and expensive procedure which requires hospitalization. The portable cardiorespiratory studies (polygraphy) have been proposed as a low cost easy to use alternative to PSG [3]. There is an ongoing debate whether polygraphic studies are suitable for OSA screening in different patient subpopulations.

The aim of our study was the assessment of polygraphic ambulatory screening in patients requiring regular tertiary care for hypertension treatment.

\section{Materials and methods}

Study population consisted of 318 patients (39\% females) recruited from the Outpatient Tertiary Care Hypertension Clinic of Medical University Hospital of Gdansk. We enrolled 243 consecutive patients regardless of the presence of OSA symptoms, as well as 75 patients with OSA suspicion referred for the sleep study by cardiovascular medicine specialists. Symptoms of sleep apnea differed between patients and were subjectively assessed by the referring physician. During the ambulatory diagnostic night, the following signals were recorded with type IV polygraphy $\left(\right.$ ApneaLink Plus ${ }^{\mathrm{T} M}$ ): airflow, snoring, pulse oximetry, chest respiratory movements. The device was operated by the user, nevertheless, subjects who required assistance with the device hook-up were provided with help during the procedure. Additionally, we ob- tained anthropometrical data, drug regimen records and selected clinical symptoms such as nocturia, and daytime sleepiness quantified with Epworth Sleepiness Scale (ESS).

\section{Statistical analysis}

All data were tabulated in MS Excel and computed in standard statistical package (Statistica v. 12, Statsoft, Poland). Continuous variables with normal distribution were compared with Student's t-test and one-way ANOVA, where appropriate. Non-normally distributed data were analyzed with non-parametric tests (Mann-Whitney). Chi-squared test was used to compare the number of cases between dichotomized subgroups. Logistic regression analysis was performed to assess the role of selected covariates with reference to diagnosed OSA. P-value less than 0.05 was considered valid for all tests.

\section{Results}

Three hundred and eight (97\%) patients completed the study. In 10 (3\%) patients, the polygraphic recording did not meet minimal evaluation criteria, thus they were excluded from further analyses (too short recordings or signal failure).

Patients were characterized by class 1 obesity $(30.0$ $\left.\pm 5.0 \mathrm{~kg} / \mathrm{m}^{2}\right)$ and body fat distribution, which suggested abdominal fat accumulation (waist circumference was $105 \pm 15.0 \mathrm{~cm}$ ). Detailed clinical characteristics of the studied group are presented in Table I.

Table I. Clinical characteristics of the study group

\begin{tabular}{|l|c|c|c|c|}
\hline & All & Random-group & Symptomatic-group & $\begin{array}{c}\text { P value (random } \\
\text { vs. symptomatic) }\end{array}$ \\
\hline Anthropometrics & & & & \\
\hline Males-to-Females ratio & $61 \%: 39 \%$ & $59 \%: 41 \%$ & $67 \%: 33 \%$ & 0.22 \\
\hline Age (years) & $57.7 \pm 11.5$ & $58.6 \pm 11.0$ & $55.1 \pm 12.7$ & 0.04 \\
\hline BMl $\left[\mathrm{kg} / \mathrm{m}^{2}\right]$ & $30.0 \pm 5.0$ & $29.5 \pm 4.6$ & $31.4 \pm 6.1$ & 0.02 \\
\hline Neck $[\mathrm{cm}]$ & $40.7 \pm 4.9$ & $39.6 \pm 4.6$ & $41.6 \pm 5.1$ & 0.01 \\
\hline Waist $[\mathrm{cm}]$ & $105.0 \pm 15.0$ & $102.4 \pm 14.3$ & $107.1 \pm 15.5$ & 0.06 \\
\hline Hips $[\mathrm{cm}]$ & $110.6 \pm 13.2$ & $108.7 \pm 9.4$ & $112.3 \pm 11.3$ & 0.04 \\
\hline No. of hypotensive drugs & $2.8 \pm 1.4$ & $2.8 \pm 1.5$ & $2.9 \pm 1.3$ & 0.65 \\
\hline Fraction of patients with $\geq 3$ hypotensive drugs & $58 \%$ & $58 \%$ & $58 \%$ & 0.97 \\
\hline Nocturia & $1.3 \pm 1.2$ & $1.3 \pm 1.3$ & $1.2 \pm 1.2$ & 0.60 \\
\hline Nocturia $>2$ & $15 \%$ & $14 \%$ & $15 \%$ & 0.87 \\
\hline Nocturia $>1$ & $38 \%$ & $40 \%$ & $36 \%$ & 0.63 \\
\hline ESS & $5.7 \pm 4.6$ & $4.4 \pm 3.6$ & $7.1 \pm 5.2$ & 0.001 \\
\hline ESS $\geq 10$ & $20 \%$ & $13 \%$ & $28 \%$ & 0.02 \\
\hline
\end{tabular}

Data presented as means $\pm \mathrm{SD} ; \mathrm{N}$ or per-cent (\%). P values for comparisons between Random- vs. Symptomatic-groups 


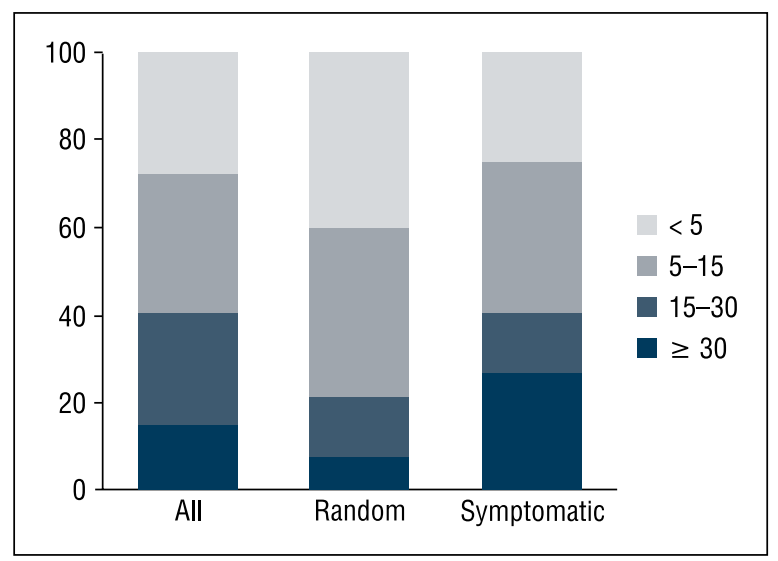

Figure 1. AHI-groups distribution depending on the presence of sleep apnea symptoms

Basic polygraphic characteristics were as follows: average AHI in the studied group was $12.8 \pm 15.2$ (10.6 \pm 11.1 vs. $19.7 \pm 22.5$ in Random vs. Symptomatic-group, respectively; $\mathrm{P}=0.01) .65 .3 \%$ of all patients were characterized by $\mathrm{AHI} \geq 5(62.2 \%$ vs. $74.7 \%$ in Random vs. Symptomatic group, respectively; $\mathrm{P}=0.49$ ). With reference to polygraphic sleep studies recommendations, low probability of sleep apnea $(\mathrm{AHI}<10)$ was present in $64 \%(65 \%$ vs. $52 \%$ in Random vs. Symptomatic group, respectively; $\mathrm{P}=0.049$ ). Twenty-seven point six percent of patients were diagnosed with AHI $\geq 15$ which corresponds to high-likelihood of sleep-apnea diagnosis and is consistent with moderate-to-severe disease based on PSG (23.6\% vs. $40.0 \%$ in Random vs. Symptomatic group, respectively; $\mathrm{P}<0.01)$. Detailed distribution of AHI-subgroups is presented in Figure 1.

$58 \%$ of all patients were treated with 3 or more hypotensive drugs. The number of administrated blood-lowering drugs varied considerably depending on AHI-group, ANOVA; P $<0.01$ (Fig. 2).

Data related to nocturia were obtained in $46 \%$ of the entire group. The distribution of nocturia severity with relation to AHI-subgroups is summarized in Figure 3. Excessive night-time voiding (>2) was associated with approximately 3.5 -fold increased odds for having been diagnosed with the highest AHI $(\geq 30)$, and adjustment to several covariates did not alter this relationship $\left(\mathrm{R}^{2}=0.29 ; \mathrm{P}<0.001\right.$, for the fully-adjusted logistic regression model (Table II, III).

Epworth Sleepiness Scale (ESS) was completed by 156 patients (50.6\%). Excessive daytime sleepiness ( $\geq 10$ pts.) was noted in $20.4 \%$ (12.8\% vs. $27.8 \%$ in Random and Symptomatic-groups, respectively; $\mathrm{P}=0.02)$.

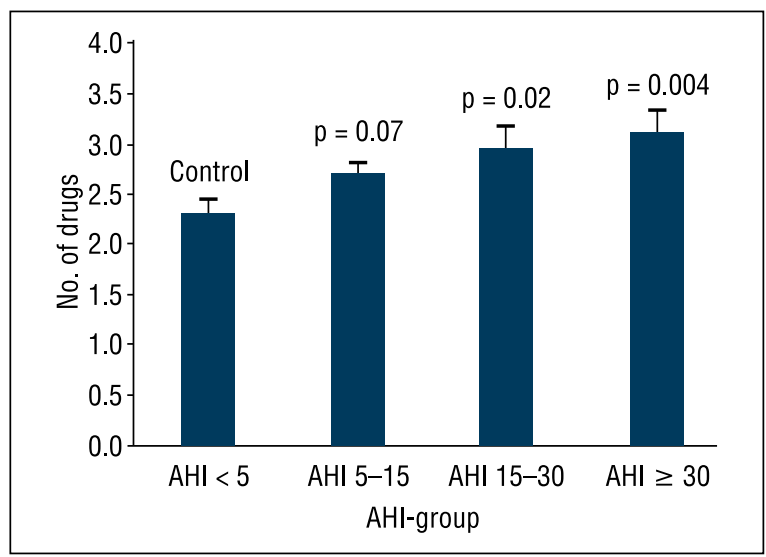

Figure 2. Blood-lowering pharmacological treatment with relation to sleep apnea severity. One-way ANOVA; $P=0.009$

Caption: Bars represent an average number of administered hypotensive drugs and whiskers standard error of the mean. P-values for post-hoc Dunnett test vs. $\mathrm{AHI}<5$ group

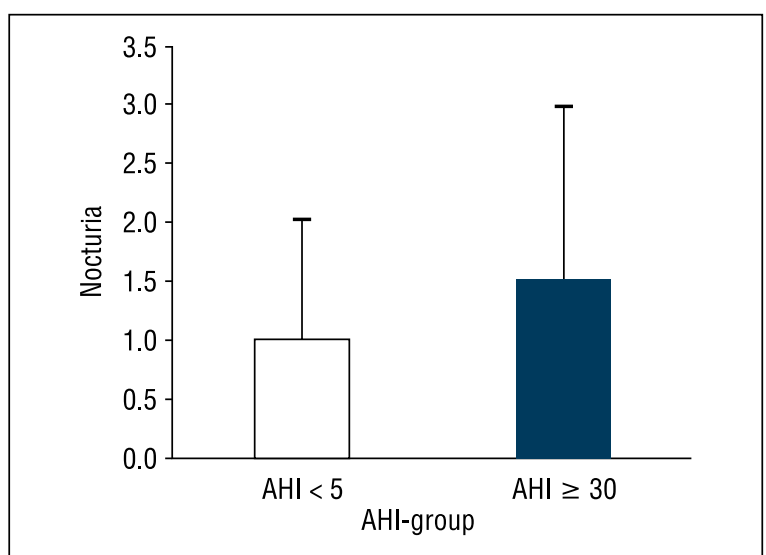

Figure 3. Number of night-time voiding with reference to $\mathrm{AHI}$ group. Mann-Whitney test, $\mathrm{P}=0.048$

Caption: Bars represent Medians and whiskers $3^{\text {rd }}$ quartile

\section{Discussion}

There are 2 main findings in our study. First, routine medical history taken in cardiovascular outpatient clinic which includes records of nocturia has the capacity to accurately identify patients with suspicion of OSA. Second, given the epidemiological burden, regular polygraphic ambulatory screening of tertiary care hypertensive patients appears to be a reasonable strategy in the comprehensive clinical management. Based on this approach, approximately one third of patients with hypertension requiring tertiary care may be eligible to OSA-treatment commencement, without further delay.

Our study supports the concept of simplified screening for OSA in the setting of tertiary care hypertension outpatient clinic. Based on the pa- 
Table II. Role of nocturia ( $>2$ times) in the prediction of severe sleep apnea. Logistic regression analysis

\begin{tabular}{|l|c|c|c|c|c|c|}
\hline & R2 & $\begin{array}{c}\text { P-value for the } \\
\text { model }\end{array}$ & OR & Lower 95\% Cl & Upper 95\% Cl & P value \\
\hline Model \#1 & 0.06 & 0.02 & 3.62 & 1.32 & 9.98 & 0.01 \\
\hline Model \#2 & 0.09 & 0.02 & 3.62 & 1.26 & 10.40 & 0.02 \\
\hline Model \#3 & 0.19 & $<0.001$ & 3.71 & 1.23 & 11.17 & 0.02 \\
\hline Model \#4 & 0.19 & 0.002 & 3.75 & 1.24 & 11.32 & 0.02 \\
\hline Model \#5 & 0.29 & $<0.001$ & 3.47 & 1.09 & 11.05 & 0.03 \\
\hline
\end{tabular}

$\mathrm{OR}$ - odds ratio

Models adjusted for:

$\# 1$ - unadjusted

$\# 2-$ nocturia $>2+$ alfa-blockers use

$\# 3$ - nocturia $>2+$ alfa-blockers use $+\mathrm{BMI}>27 \mathrm{~kg} / \mathrm{m}^{2}$

$\# 4$ - nocturia $>2+$ alfa-blockers use $+\mathrm{BMI}>27 \mathrm{~kg} / \mathrm{m}^{2}+$ age $>60$ years old

$\# 5$ - nocturia $>2+$ alfa-blockers use $+\mathrm{BMl}>27 \mathrm{~kg} / \mathrm{m}^{2}+$ age $>60$ years old + male sex

Table III. Odds ratios for the fully adjusted model (Model No. 5). Logistic regression analysis $\mathrm{R}^{2}=0.29 ; \mathrm{P}<0.001$

\begin{tabular}{|l|c|c|c|c|}
\hline & OR & Lower 95\% Cl & Upper 95\% Cl & P value \\
\hline Nocturia (> 2) & $\mathbf{3 . 4 7}$ & 1.09 & 11.05 & 0.03 \\
\hline Alpha-blockers use & 1.13 & 0.37 & 3.48 & NS \\
\hline Male sex & 9.21 & 1.76 & 48.17 & 0.01 \\
\hline BMl greater than $27 \mathrm{~kg} / \mathrm{m}^{2}$ & 9.04 & 1.12 & 73.84 & 0.04 \\
\hline Age greater than 60 years old & 1.83 & 0.59 & 5.67 & NS \\
\hline
\end{tabular}

$O R$ - odds ratio

tient-operated, ambulatory cardiorespiratory polygraphic recordings, approximately half of the tertiary-care hypertensive population is characterized by low probability of OSA, conversely, one third of them is eligible for the introduction of OSA treatment without any further delay. This percentage may be further increased by complementary full polysomnographic recordings, as the polygraphic studies, by definition, are characterized by approximately $30 \%$ lower respiratory indices [4]. Additionally, abridged medical history which is focused on selected sleep apnea symptoms may substantially increase the accuracy of OSA-patients identification.

\section{Nocturia}

Episodes of night-time voiding disrupt sleep continuity which in turn worsens sleep quality, and may affect patients' daytime performance. Nocturia, the symptom which is rarely associated with OSA in everyday clinical and ambulatory practice, appears to be a very potent marker of undiagnosed sleep disordered breathing. Commonly, nocturia is associated with several chronic medical conditions in adult population such as heart failure, benign prostate hyperplasia, or diabetes mellitus, however, OSA is rarely taken under consideration in the differential diagnosis. Interestingly, recent analyses identify nocturia as an independent predictor of all-cause mortality [5].
Nocturia may reflect the severity of sleep apnea [6] and it may also be ameliorated by OSA reversal (by means of continuous positive airway pressure; $\mathrm{CPAP}$ ) [7, 8]. Our data showed that night-time voiding greater than 2 times is associated with the increased odds for the presence of undiagnosed severe sleep apnea in a random group of patients who regularly attend tertiary care out-patient hypertension clinic. In different logistic regression models, nocturia $(>2)$ increased the odds of AHI $\geq 30$ diagnosis about 3.5 times (Table II). Interestingly, the adjustment for different covariates (including alfa-blocker use which itself may considerably affect severity of nocturia) did not substantially alter the outcome (Table II and III).

\section{Hypertension control}

By definition, patients requiring 3 or more blood-lowering drugs including diuretic are considered drug-resistant to standard treatment $[9,10]$. Although the prevalence of such condition is usually difficult to estimate precisely (up to $15 \%$ depending on the subpopulation assessed) [11], the presence of truly resistant hypertension is relatively low and closely depends on patients' compliance. In our cohort, $58 \%$ of screened patients were treated with 3 or more hypotensive drugs (Table I). This may not be directly translated to resistant hypertension diagnosis, nevertheless it appears to be a very high 
rate even for patients requiring tertiary care. Such phenomenon may be partly explained by two main issues. First, following the recommendation for the fixed-dose therapies, tertiary care physicians are more likely to administer such formulas. Second, there is a considerable number of patients who were previously undiagnosed with sleep apnea, which may conceivably add to their blood pressure load. We report that about $40 \%$ of patients who are regular attendants of our Hypertension Centre may be diagnosed with moderate-to-severe disease (Fig. 1). All of the hypertension management guidelines issued in the last decade underscored the need for sleep-disordered breathing screening and treatment in almost every patient evaluated for blood-lowering treatment failure $[1,10,12,13]$. As there is an incremental relationship between severity of apnea and the risk for hypertension observed in the general population $[14,15]$, also severity of OSA may contribute to blood-lowering treatment difficulties in hypertensives [16]. Based on our clinical data, we showed that patients with $\mathrm{AHI} \geq 30$ require significantly higher number of hypotensive drugs comparing to their counterparts (Fig. 2), and the presence of OSA symptoms did not alter this relationship (Table I). This finding underscores wide screening of sleep apnea even in minimally symptomatic patients. Given the fact, that CPAP treatment considerably modifies the discussed association [17], as well as there is an evident CPAP-related blood pressure lowering effect [18], a prompt OSA screening in corresponding populations should be endorsed.

\section{Obesity}

The role of obesity in the development of sleep-disordered breathing has been well established [19]. In our cohort, average BMI for the entire study group equaled $30.0 \pm 5.0 \mathrm{~kg} / \mathrm{m}^{2}$ which is consistent with class 1 obesity diagnosis. There was a difference in BMI recorded in Random vs. Symptomatic groups: $29.5 \pm 4.6$ vs. $31.4 \pm 6.1 \mathrm{~kg} / \mathrm{m}^{2}$, respectively (Table I). Although the mean difference in BMI between the two subgroups was rather modest (approx. $2 \mathrm{~kg} / \mathrm{m}^{2}$ ), the logistic regression pointed at the assessment of the influence of different covariates on the development of sleep apnea further confirmed its independent role (BMI $>27 \mathrm{~kg} / \mathrm{m}^{2}$ was associated with increased odds for the diagnosis of AHI $\geq 30$ by approx. 9 times) (Table III). It is worth mentioning that American guidelines on hypertension management (JNC7) issued more than a decade ago stressed the role of an increased BMI as an accurate indicator for suspected OSA in the hypertensive population [1]. Experts of JNC7 recommended thorough screening for OSA, even in relatively lean hypertensive patients (BMI greater than $27 \mathrm{~kg} / \mathrm{m}^{2}$ which is consistent with the diagnosis of overweight). In line with JNC 7, recent guidelines of the Polish Society of Hypertension (PTNT) also characterized hypertensives requiring further diagnosis of sleep-disordered breathing. Of more, the metabolic syndrome, a condition mainly determined by comorbid obesity is emphasized by PTNT as a valuable pre-screening tool in OSA-suspicion [10].

\section{Strengths and limitations}

Our study did not aim at comparing of the polygraphic recordings vs. attended polysomnography. However, it was shown elsewhere that portable devices fairly reflect the PSG results, accurately stratify patients [20-22], as well as they are sufficiently reliable as for the decision on CPAP treatment commencement [23]. There are unquestionable advantages of ambulatory screening over attended PSG. Most important such procedure does not require adaptation night as patients sleep in their natural environment. Sleep societies endorse screening for OSA with polygraphic devices only in selected subpopulations [24], however, cardiovascular entities are more likely to support its use as an effective pre-screening tool in a wide spectrum of CV-patients [10]. As we observed screening rate $(\mathrm{AHI} \geq 5)$ in more than $70 \%$ of hypertensive patients, our data support such strategy. Nevertheless, it is critical to remember that portable monitoring devices have the capacity to confirm OSA, but are not sufficient to exclude any of the sleep-disordered breathing, especially in patients complaining of OSA-symptoms.

\section{Conclusions}

We conclude that frequent nocturia may serve as a potent marker of undiagnosed severe sleep apnea. Our data also support the concept of ambulatory polygraphic screening for OSA even in minimally symptomatic patients requiring hypertension tertiary care.

\section{References}

1. Chobanian A.V., Bakris G.L., Black H.R. et al. The Seventh Report of the Joint National Committee on Prevention, Detection, Evaluation, and Treatment of High Blood Pressure: the JNC 7 report. JAMA 2003; 289: 2560-2572.

2. Logan A.G., Perlikowski S.M., Mente A. et al. High prevalence of unrecognized sleep apnoea in drug-resistant hypertension. J. Hypertens. 2001; 19: 2271-2277.

3. Masa J.F., Corral J., Pereira R. et al. Effectiveness of sequential automatic-manual home respiratory polygraphy scoring. Eur. Respir. J. 2013; 41: 879-887. 
4. Escourrou P., Grote L., Penzel T. et al. The diagnostic method has a strong influence on classification of obstructive sleep apnea. J. Sleep Res. 2015; 24: 730-738.

5. Fan Y., Wei F., Lang Y., Qi W. Meta-analysis of nocturia and risk of all-cause mortality in adult population. Int. J. Cardiol. 2015; 195: 120-122.

6. Oztura I., Kaynak D., Kaynak H.C. Nocturia in sleep-disordered breathing. Sleep Med. 200;, 7: 362-367.

7. Miyazaki T., Kojima S., Yamamuro M. et al. Nocturia in Patients With Sleep-Disordered Breathing and Cardiovascular Disease. Circ. J. 2015; 79: 2632-2640.

8. Wang T., Huang W., Zong H., Zhang Y. The Efficacy of Continuous Positive Airway Pressure Therapy on Nocturia in Patients With Obstructive Sleep Apnea: a Systematic Review and Meta-Analysis. Int. Neurourol. J. 2015; 19: 178-184.

9. Calhoun D.A., Jones D., Textor S. et al. Resistant Hypertension: Diagnosis, Evaluation, and Treatment. Circulation. 2008; 117: e $510-526$.

10. Tykarski A., Narkiewicz K., Gaciong Z. et al. 2015 guidelines for the management of hypertension. Recommendations of the Polish Society of Hypertension — short version. Kardiol. Pol. 2015; 73: 676-700.

11. Siddiqui M., Dudenbostel T., Calhoun D.A. Resistant and Refractory Hypertension: Antihypertensive Treatment Resistance vs Treatment Failure. Can. J. Cardiol. 2015 Jul 8. pii: S0828-282X(15)00515-2. doi: 10.1016/j.cjca.2015.06.033. [Epub ahead of print]

12. Mancia G., Fagard R., Narkiewicz K. et al. 2013 ESH/ESC Guidelines for the management of arterial hypertension: the Task Force for the management of arterial hypertension of the European Society of Hypertension (ESH) and of the European Society of Cardiology (ESC). J. Hypertens. 2013; 31: 1281-1357.

13. Parati G., Lombardi C., Hedner J. et al. Position paper on the management of patients with obstructive sleep apnea and hypertension: joint recommendations by the European Society of Hypertension, by the European Respiratory Society and by the members of European COST (COoperation in Scientific and Technological research)
ACTION B26 on obstructive sleep apnea. J. Hypertens. 2012; 30: 633-646.

14. Peppard P.E., Young T., Palta M., Skatrud J. Prospective study of the association between sleep-disordered breathing and hypertension. N. Engl. J. Med. 2000; 342: 1378-1384.

15. Lavie P., Herer P., Hoffstein V. Obstructive sleep apnoea syndrome as a risk factor for hypertension: population study. BMJ 2000;320:479-482.

16. Walia H.K., Li H., Rueschman M. et al. Association of severe obstructive sleep apnea and elevated blood pressure despite antihypertensive medication use. J. Clin. Sleep. Med. 2014; 10: 835-843.

17. Marin J.M., Agusti A., Villar I. et al. Association between treated and untreated obstructive sleep apnea and risk of hypertension. JAMA 2012; 307: 2169-2176.

18. Tsioufis C., Kasiakogias A., Thomopoulos C., Manolis A., Stefanadis C. Managing hypertension in obstructive sleep apnea: the interplay of continuous positive airway pressure, medication and chronotherapy. J. Hypertens. 2010; 28: 875-882.

19. Young T., Shahar E., Nieto F.J. et al. Predictors of sleep-disordered breathing in community-dwelling adults: the Sleep Heart Health Study. Arch. Intern. Med. 2002; 162: 893-900.

20. Ng S.S., Chan T.O., To K.W. et al. Validation of a portable recording device (ApneaLink) for identifying patients with suspected obstructive sleep apnoea syndrome. Intern. Med. J. 2009; 39: 757-762.

21. Lesser D.J., Haddad G.G., Bush R.A., Pian M.S. The utility of a portable recording device for screening of obstructive sleep apnea in obese adolescents. J. Clin. Sleep. Med. 2012; 8: 271-277.

22. Weinreich G., Armitstead J., Topfer V., Wang Y.M., Wang Y., Teschler H. Validation of ApneaLink as screening device for Cheyne-Stokes respiration. Sleep 2009; 32: 553-557.

23. Douglas N.J. Home diagnosis of the obstructive sleep apnoea/hypopnoea syndrome. Sleep Med. Rev. 2003; 7: 53-59.

24. Collop N.A., Anderson W.M., Boehlecke B. et al. Clinical guidelines for the use of unattended portable monitors in the diagnosis of obstructive sleep apnea in adult patients. Portable Monitoring Task Force of the American Academy of Sleep Medicine. J. Clin. Sleep Med. 2007; 3: 737-747. 